\title{
Blood Typing and Twin Zygosity: A Comparison of Two Methods
}

\author{
David T. Lykken \\ Psychiatry Research Unit, University of Minnesota, Minneapolis
}

In a recent paper in this journal [5], Wilson offers a revision of his previously published method [4] for diagnosing the zygosity of twins from bloodtype findings. Wilson notes that his revised method will yield the same results (given the same estimates of gene frequencies) as will the methods described by Smith and Penrose [2], Sutton et al [3], and Lykken [1], and he advocates his new method, over the others, as being "simplest and most direct." He also reinterprets various quantities employed by Lykken and proposes a different index of the efficiency of a genetic marker for zygosity diagnosis. In this note, I attempt to clarify the relationship between Wilson's revised method and my own, so that the reader can more accurately assess their relative advantages.

Key words: Twins, Blood types, Zygosity

\section{ZYGOSITY DIAGNOSIS FROM MENDELIZING TRAITS}

Barring somatic mutations, a twin pair that is discordant on any bloodgroup or other Mendelizing trait must be dizygotic (DZ). One needs a "method" only for the case where the twin pair is concordant for all $k$ traits that have been assessed. The purpose of the method is to allow one to estimate the residual probability that the pair is DZ in the face of this concordance. This will require a knowledge of the relative frequencies of all relevant genes in the population from which the twins were drawn as well as an understanding of the genetic mechanism for each of the $k$ traits. Step 1 of any zygosity diagnosis method will employ this genetic information to compute some type of estimate of the likelihood that DZ twins would be concordant on each of the possible traits. Ideally, these estimates will be tabulated so that the user can merely look up the values needed for a particular twin pair. Step 2 of the method will involve combining the $k$ individual likelihood estimates into a single conditional probability, $\mathrm{P}(\mathrm{DZ} \mid \mathrm{C})$, which is the probability

This research was supported by a grant from the National Institute of Mental Health. 
that the given pair, concordant for the particular array of phenotypes, is nevertheless DZ rather than $\mathrm{MZ}$.

Smith and Penrose [2], Sutton et al [3], and Lykken [1] all calculate in step 1 the conditional probability $\mathrm{P}\left(\mathrm{C}_{\mathrm{i}} \mid \mathrm{DZ}\right)$, which is the probability that a pair of $\mathrm{DZ}$ twins, at least one of whom shows the $i$ th phenotype, will be concordant for that phenotype. This quantity can also be interpreted as the odds that the pair is $\mathrm{DZ}$ rather than $\mathrm{MZ}$, given they are concordant for $i$, as is shown by the following relationship:

$$
\operatorname{Odds}_{\mathrm{DZ} \mid \mathrm{C}_{i}}=\mathrm{P}\left(\mathrm{C}_{\mathrm{i}} \mid \mathrm{DZ}\right) / \mathrm{P}\left(\mathrm{C}_{\mathrm{i}} \mid \mathrm{MZ}\right)=\mathrm{P}\left(\mathrm{C}_{\mathrm{i}} \mid \mathrm{DZ}\right)
$$

In step 2, these individual values are multiplied together to yield the odds that the twins are DZ given that they are concordant on the entire array of phenotypes, ie, the Odds $_{\mathrm{DZ} \mid \mathrm{C}}$. This value is then multiplied by the initial odds that any same-sex twin pair will be $\mathrm{DZ}$ (about 0.85 ). In Lykken's method, measures of within-pair similarity on various polygenic traits, such as fingerprint ridge count, provide additional values that can be used to further increase the total odds against dizygosity. Because one is accustomed to thinking in terms of probabilities rather than odds, this final value can be converted to the probability that this pair is DZ by the relation:

$$
\mathrm{P}(\mathrm{DZ} \mid \mathrm{C})=\operatorname{Odds}_{\mathrm{DZ} \mid \mathrm{C}} /\left(1+\operatorname{Odds}_{\mathrm{DZ} \mid \mathrm{C}}\right)
$$

In step 1 Wilson computes two values instead of one for each phenotype. The first, which he calls the "one zygote probability," is the conditional probability $\mathrm{P}(\mathrm{MZ} / \mathrm{CM})$ where CM refers to a mating capable of producing the $i$ th phenotype; this quantity is equal to the probability that, among MZ twins that might be produced by such a capable mating (CM), a particular pair of $\mathrm{MZ}$ twins will show the $i$ th phenotype. Wilson also tabulates a second value, the "two zygote probability," which is the conditional probability $\mathrm{P}\left(\mathrm{DZ} \mathrm{Z}_{\mathrm{i}} \mid \mathrm{CM}\right)$, the probability that a pair of $\mathrm{DZ}$ twins, produced by this same capable mating, will be concordant in $i$. Wilson's step 2 involves multiplying together the $k$ one-zygote probabilities, the $k$ two-zygote probabilities, and then combining them with the prior probabilities that same-sex twins are $M Z$ or $D Z$ in a Bayesian formula to yield the desired value of $\mathrm{P}(\mathrm{DZ} \mid \mathrm{C})$. This is only slightly more laborious than Lykken's step 2 and will yield the correct result.

\section{WILSON VS LYKKEN/SUTTON ET AL}

As Wilson acknowledges, step 1 of his method is “. . . cumbersome since [it requires] the complete enumeration of all possible mating combinations and all possible pairs of offspring within each bloodgroup." [5: p 107]. In this respect it resembles the method of Smith and Penrose and is in marked contrast to Lykken's step 1, which employs the formulas provided by Sutton et al [5]. For practical purposes, an investigator who elects Wilson's method is restricted to the eight bloodgroup systems that Wilson tabulates and is restricted also to the particular estimates of gene frequencies on which those tables are based. Lykken [1] tabulates the same eight bloodgroups plus ten serum proteins and red blood cell enzymes that are now commonly available in serology studies, plus three independent polygenic variables (ponderal index, cephalic index, and Slater's Z score based on fingerprint comparisons). As explained [1: pp 472-473], the expected value of 
$\mathrm{P}(\mathrm{DZ} \mid \mathrm{C})$ associated with the average pair of $\mathrm{MZ}$ twins tested on just the eight bloodgroups will be about 0.018 . Using all 21 variables, the equivalent value is 0.00031 .

More important than the number of markers tabulated in the two articles, however, is the relative ease of calculating the needed statistics on new variables that an investigator might wish to employ, or recalculating the statistics on the tabled variables using gene frequency estimates that may be more appropriate to the population from which the investigator's twin sample was drawn. Because Wilson's method is "cumbersome," four pages of appendix, including four tables, are required just to illustrate the step 1 calculations for the two phenotypes of the simple $P$ bloodgroup system. If one were dealing with, say, a pair of black twins for whom Wilson's tables are inappropriate due to different gene frequencies in white and black populations, recomputing his statistics with the correct gene frequencies would be a task not lightly undertaken. Using Lykken's method and the formulae of Slater et al, the same task can be accomplished in ten minutes with a hand calculation.

\section{REFERENCES}

1. Lykken, DT (1978): The diagnosis of zygosity in twins. Behav Genet 8:437-473.

2. Smith, SM, Penrose, LS (1955): Monozygotic and dizygotic twin diagnosis. Ann Hum Genet 19:273-289.

3. Sutton, HE, Clark, PJ, Schull, WJ (1955): The use of multi-allele genetic characters in the diagnosis of twin zygosity. Am J Hum Genet 7:180-188.

4. Wilson, RC (1970): Bloodtyping and twin zygosity. Hum Hered 20:30-56.

5. Wilson, RS (1980): Bloodtyping and twin zygosity: Reassessment and extension. Acta Genet Med Gemellol 29:103-120.

Correspondence: D.T. Lykken, Psychiatry Research Unit, Box 392, Mayo, University of Minnesota, Minneapolis, MN 55455, USA. 